Research Article

\title{
Networked Base-Stock Inventory Control in Complex Distribution Systems
}

\author{
Przemysław Ignaciuk (i) and Lukasz Wieczorek (D) \\ Institute of Information Technology, Lodz University of Technology, 215 Wólczańska St, 90-924 Łódź, Poland \\ Correspondence should be addressed to Przemysław Ignaciuk; przemyslaw.ignaciuk@p.lodz.pl
}

Received 27 June 2019; Revised 25 July 2019; Accepted 29 August 2019; Published 4 November 2019

Academic Editor: Mauro Gaggero

Copyright (c) 2019 Przemysław Ignaciuk and Łukasz Wieczorek. This is an open access article distributed under the Creative Commons Attribution License, which permits unrestricted use, distribution, and reproduction in any medium, provided the original work is properly cited.

\begin{abstract}
This paper addresses the resource distribution problem in logistic networks with a complex, multi-echelon interconnection structure. The considered class of systems encompasses two types of nodes: controlled nodes subject to inventory management decisions and sources providing goods to the system to sustain the sales. The stock gathered at the controlled nodes is used to satisfy an exogenous, uncertain demand that may be placed at any controlled node. The stock is replenished from the sources and other controlled nodes, and the flow of goods proceeds with positive lead-time delay. The network topology does not assume any structural simplifications typically considered in inventory control problems, i.e., the nodes can form a link with any other node which leads to a mesh, multi-echelon structure. The contribution of this paper is twofold: First, the classical order-up-to policy is analyzed formally with respect to such nontrivial connectivity architectures, and conditions for full demand satisfaction under any bounded demand pattern are established. Next, a networked extension of the classical policy is proposed. The introduced networked policy is shown to generate smaller holding costs while maintaining the same service level as the classical policy. The formal study is supported by numerical tests involving genetic algorithms and simulation-based optimization.
\end{abstract}

\section{Introduction}

In recent years, the observed tight internationalization and development of global market have caused changes in many sectors of the economy. New mechanisms and processes are being introduced on a daily basis, and the existing ones undergo transformations and rescaling. In particular, the now commonly available intelligent transportation, communication, and information system (IT) enhancements $[1,2]$ exert substantial impact on the practices in logistics and may reshape the entire sector related to the management of the flow of goods and execution of services in distributed environments [3-6]. However, owing to unforeseeable factors and uncertain operational conditions [7] combined with ever growing quality requirements, the research on structurally complex supply chains and networks faces difficulties in both analytical and computational treatment. So far, the literature has been centered on single-echelon [7-10], two-stage serial [11-13], and multi-echelon arborescent configurations [14-16], which are simplifications of the actual architectures presently deployed [17]. The most challenging aspect of more complex topologies is the dynamical interdependencies that lead to multidimensional, nonseparable space of solutions. Affected by nonlinearities and nonnegligible lead times, the networked distribution systems are not amenable to the popular methods proved efficient in simpler architectures, e.g., Markov chain-based approach [18]. As an attractive alternative, one may consider dynamical system approach [19], if well adjusted for the challenging fullconnectivity (mesh) topologies.

1.1. Dynamic System Approach in Inventory Control. The pioneering exposition in the systemic approach to inventory control problems is usually attributed to Forrester [20], who used coupled nonlinear differential equations to capture the dynamics of supply chains. In order to address the ensuing analytical intricacies, a number of simplified models, 
adopting, principally, the frequency domain approach, have been introduced by various researchers by the end of the $20^{\text {th }}$ century [21-23]. These models have later been extended to cover the key effects of delay, in either continuous-time framework [24, 25] or discrete-time framework [26-29]. In turn, the multinode interaction was studied using primarily the state-space approach [30-34], where the emphasis was placed on formal design aspects under various, not necessarily economic, optimization objectives. A summary of previous developments adopting the dynamic system approach has been provided in the excellent review papers by Ortega and Lin [35], Sarimveis et al. [36], and the book by Axsäter [37], whereas the account of current results is given by Zemzam et al. [38] and Ivanov et al. [39].

A significant limitation of the majority of earlier models is assuming linear dynamics in the operational region of interest in order to exercise the formal approach. For instance, a full-backordering restriction has been enforced to avoid the nonlinearities associated with the otherwise saturating stock function required in the lost-sales systems in [26]. On the other hand, the models with explicit capacity constraints incorporated rarely accept a closed-form solution, and only in the simplified case of serial, or fully separable paths of goods flow [40]. In the cases of practical significance, e.g., under demand uncertainty [41] and leadtime variations [42-44], the researchers rely on the numerical analysis. Recently, e.g., Danielson et al. [45] and Bürger et al. [46] address more complete connectivity structures (modeled through the incidence matrix) for general resource distribution networks. Two objectives-achieving balanced network state while responding to the local demand and system capacity optimization-have been considered and attained through (numerical) solution of the related optimization problem. However, the issues of delay in resource transshipment were ignored, neither robustness aspects were covered. More complex models are typically handled through simulation only [47, 48], which limits generalization of managerial insights.

Despite intensified effort [49], multi-echelon production and goods distribution systems require further study. In particular, the issues of delay and demand uncertainty in the systems with arbitrary topological configuration need more attention while assessing the performance of classical control policies, executing tuning procedures, or building new design paradigms.

1.2. Objectives and Paper Contribution. One of the basic premises of a well-conditioned production-inventory system is to fulfill the demand, internal originating from the neighboring entities in the goods distribution structure-or exogenous-placed by external actors. In the majority of real-life cases, the demand is not known a priori and may fluctuate with time in an uncertain way, which poses a significant challenge for balancing the costs and revenues. Besides logistics, this uncertainty is encountered in other contexts as well, i.e., trade [50], tourism [51], or energy distribution [52]. In order to address its negative implications, various resource management strategies are proposed and tuned to the specifics of a particular problem. In the network setting, the optimization objectives typically stress two aspects. On the one hand, it is desirable to reduce the operational costs, notably, the holding costs related to goods storage at the network nodes. On the other hand, one wishes to secure a high level of customer satisfaction, which, besides economic benefits, aims at strengthening the company credibility on the market.

Following the robust control convention from Sarimveis et al. [36], in this work, the demand is modeled as an arbitrary-bounded function of time. The goods distribution process proceeds in a network of nodes connected in a mesh topology, i.e., there is no structural restriction concerning the node linkage except for the basic assumption of reachability of every node. The demand can be placed at any node, and the resource reflow is subject to positive lead time, possibly different with respect to each internode link. The stock gathered at the controlled nodes-to respond to the external demand and satisfy the internal traffic-is replenished from goods sources. Again, there is no restriction as to which nodes are directly connected with the sources. The objective of this paper is twofold:

(1) To investigate analytically the performance of the classical base-stock (CBS) policy, implemented in a distribution way (independently at each node), in the considered general class of logistic systems

(2) To examine how the information about the network topological structure can assist in taking more judicious ordering decisions via the application of a networked base-stock (NBS) strategy, developed using formal, control-theoretic approach

This paper is organized in the following way. The system model and its state-space form are introduced in Section 2. The ordering policies, put in the proposed framework, and the detailed analytical treatment are also given in Sections 2. Section 3 is devoted to numerical study. Finally, conclusions and managerial insights are presented in Section 4 . The main symbols and quantities used in the text are grouped in Table 1.

\section{Analytical Framework}

2.1. Preliminaries. Let us consider the goods distribution network, comprising five nodes, depicted in Figure 1. Nodes 1-3 constitute the controlled elements subject to the ordering decisions, whereas nodes 4 and 5 are the sources used to replenish the stock inside the three-node network. Nodes 1 and 2, in addition to responding to the requests from a local market according to demands $d_{1}$ and $d_{2}$, respectively, serve as intermediate suppliers for node 3 . Node 3 may be perceived as the distribution center providing goods for other markets according to demand $d_{3}$. The arrows in the graph in Figure 1 indicate the flow of goods and the pair of numbers-the fraction of order issued by a node for its neighbor and the delay in procuring the goods from the neighbor, respectively. Although simple from the topological perspective, the considered network displays two particularities that make the search for an optimal 
TABle 1: Applied notation.

\begin{tabular}{|c|c|}
\hline Symbol & Definition \\
\hline$t \in\{1,2, \ldots, T\}$ & Time period, $T$-planning horizon \\
\hline$i \in\{1,2, \ldots, N\}$ & $\begin{array}{c}\text { Index of a controlled node, } N \text {-number of } \\
\text { controlled nodes }\end{array}$ \\
\hline$j \in\{1,2, \ldots, M\}$ & $\begin{array}{c}\text { Index of a source of goods, } M-\text { number of } \\
\text { sources }\end{array}$ \\
\hline$P=N+M$ & Total number of nodes in the network \\
\hline$\Theta_{i}$ & $\begin{array}{l}\text { Index set of suppliers for controlled node } i \\
\text { Lead-time delay of procuring an order from node }\end{array}$ \\
\hline$\tau_{j i}$ & $\begin{array}{l}j \text { to } i \text {, comprising preparation and transportation } \\
\text { time } \tau_{j i}=\tau_{j}^{\text {prep }}+\tau_{j i}^{\text {trans }} ; \tau_{\max }-\text { the maximum lead } \\
\text { time at any internode link }\end{array}$ \\
\hline$\varphi_{j i}$ & $\begin{array}{l}\text { Supplier contribution-the part of order } \\
\text { generated by node } i \text { assigned for its supplier } j\end{array}$ \\
\hline$x_{i}(t)$ & On-hand stock level at node $i$ in period $t$ \\
\hline$d_{i}(t)$ & External demand imposed on node $i$ in period $t$ \\
\hline$h_{i}(t)$ & Satisfied demand at node $i$ in period $t$ \\
\hline$q_{i}(t)$ & $\begin{array}{l}\text { Quantity of goods requested by node } i \text { as the } \\
\text { replenishment order in period } t\end{array}$ \\
\hline$u_{i}(t)$ & $\begin{array}{l}\text { Quantity of goods received by node } i \text { from its } \\
\text { suppliers in period } t\end{array}$ \\
\hline$o_{i}(t)$ & $\begin{array}{c}\text { Quantity of goods sent by node } i \text { to neighbors in } \\
\text { period } t\end{array}$ \\
\hline $\mathbf{x}(t)$ & $\begin{array}{l}\text { Vector grouping on-hand stock levels, } \mathbf{x}(t)= \\
{\left[x_{1}(t), \ldots, x_{N}(t)\right]^{\prime}}\end{array}$ \\
\hline $\mathbf{q}(t)$ & $\begin{array}{l}\text { Vector grouping ordering signals, } \mathbf{q}(t)=\left[q_{1}(t),\right. \\
\left.\qquad \ldots, q_{N}(t)\right]^{\prime}\end{array}$ \\
\hline $\mathbf{d}(t)$ & $\begin{array}{l}\text { Vector grouping market demands imposed on } \\
\text { the controlled nodes, } \mathbf{d}(t)=\left[d_{1}(t), \ldots, d_{N}(t)\right]^{\prime}\end{array}$ \\
\hline$[\cdot]^{\prime}$ & Transpose operator \\
\hline
\end{tabular}

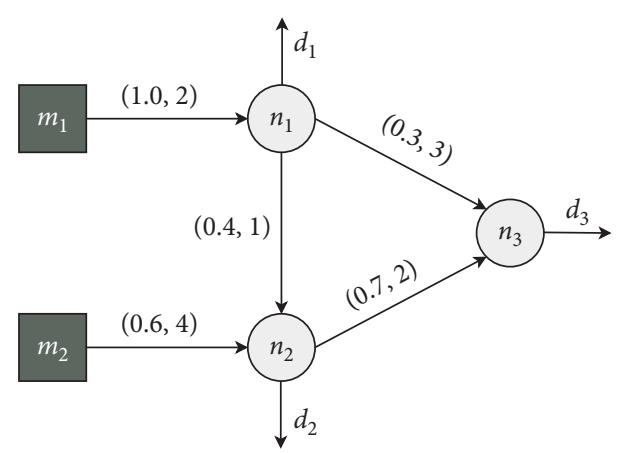

FIGURE 1: Five-node network: $n_{1}, n_{2}$, and $n_{3}$ indicate controlled nodes, $m_{1}$ and $m_{2}$ indicate external sources, and $d_{1}, d_{2}$, and $d_{3}$ denote market demand.

policy a cumbersome task when faced by an uncertain demand. First of all, the demand is placed at any node, i.e., not just conveniently at the distribution center (node 3 ). Secondly, the connection structure cannot be disentangled into separate paths converging at a focal point owing to the link between nodes 1 and 2, as is typically considered in multi-echelon systems [40]. According to Cattani et al. [53], any precarious topological simplification may lead to erroneous decisions and serious cost increase.

2.2. Fundamental Assumptions. The considered class of logistic networks assumes cooperation among three types of entities: goods sources, controlled nodes subject to managerial decisions, and customers generating demand. The purpose of the goods distribution system is to satisfy the (uncertain) market demands imposed on the controlled nodes without recurring to excessive safety stock. The flow of goods among the nodes is realized through unidirectional channels that form a mesh interconnection topology, i.e., the network organization does not imply any restriction as to which nodes are connected with each other, except for the impractical cases of isolated entities (without a path to any source), or a node supplying goods to itself.

Each node interconnection is characterized by two parameters: supplier contribution and lead-time delay. The supplier contribution specifies the part of order issued by a controlled node that is to be acquired from the chosen supplier (a neighboring controlled node in the network, or a source). The lead-time delay covers the time of all the activities that are related to procuring the order, e.g., preparation, loading/unloading, shipment, and inspection.

The market demands are not known a priori and do not take any constraints with respect to the stochastic or deterministic variation pattern, except they are all nonnegative and bounded from above. They may be imposed on any controlled node and at any time during the planning horizon of $T$ equally spaced time periods $0,1, \ldots, t, \ldots, T$.

2.3. System Model. The sequence of operations performed at a controlled node in each period proceeds according to the diagram depicted in Figure 2. First, the incoming shipments from its suppliers are registered into stock. Next, the node tries to satisfy the external demand placed by customers. Unsatisfied demand is lost. Finally, if the stock is sufficient, the goods are sent to the neighbors in the networkrequesting goods via internal replenishment orders. Hence, a higher priority is given to satisfying the customer demand over the internal resource distribution.

2.3.1. Incoming Shipments. Let $q_{j i}(t)$ denote the goods quantity requested by node $i$ from node $j$ related to the aggregate replenishment order $q_{i}(t)$ generated in period $t$, and $o_{j i}(t)$ be the shipment actually sent by node $j$ to $i$ according to such request generated $\tau_{j i}$ periods earlier. $\tau_{j i}$ represents the nonnegligible time it takes to transfer the goods between any two neighbors. The amount of incoming goods at node $i$, with respect to supplier $j$, is given by the following equation:

$$
u_{j i}(t)=o_{j i}\left(t-\tau_{j i}\right) .
$$

And, the overall goods quantity received by node $i$ in period $t$ is given by

$$
u_{i}(t)=\sum_{j \in \Theta_{i}} o_{j i}\left(t-\tau_{j i}\right),
$$

where $\Theta_{i}$ is the index set of the suppliers of node $i$. Since in the model, satisfying the external demand at the controlled nodes takes precedence over internal shipments, the amount of goods received from a neighbor, $u_{j i}(t)$, may be smaller 


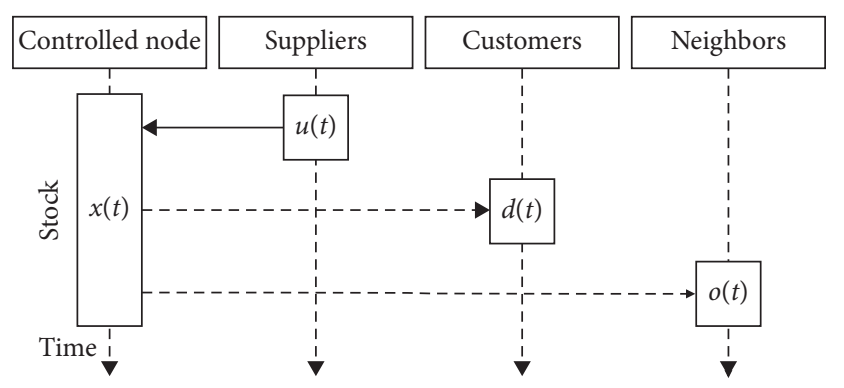

FIgURE 2: Sequence of operations at a controlled node.

than the amount requested $q_{j i}\left(t-\tau_{j i}\right)$, except the case when the supplier is an external source-the external sources are assumed uncapacitated. Thus, one has the relation

$$
0 \leq \sum_{j \in \Theta_{i}} u_{j i}(t) \leq q_{i}\left(t-\tau_{j i}\right) \text {. }
$$

2.3.2. Outgoing Shipments. Node $j$ (in its role of goods supplier) attempts to fulfill all the replenishment requests $q_{j i}$ placed by its neighbors. Let $\varphi_{i j} \in[0,1], \sum_{j \in \Theta_{i}} \varphi_{j i}=1$, denote the nominal supplier contribution in the relation between nodes $i$ and $j$. (Obviously, $\varphi_{i j}=0$ if nodes $i$ and $j$ do not form a neighboring relationship.) The amount of goods requested by node $i$ from node $j$ is given by

$$
q_{j i}(t)=\varphi_{j i} q_{i}(t)
$$

However, after satisfying the external demand, and in the absence of sufficient on-hand stock, the amount of goods actually sent to the neighbors is lower than the quantity requested. In such a case-goods shortage at node $j$-the replenishment request issued by node $i$ is decreased (in proportion to the load generated by all the neighbors of node $j)$. The reduction is reflected in the change of source contribution from $\varphi_{j i}$ to $\varphi_{j i}(t)<\varphi_{j i}$. Consequently, the quantity of goods in the internal shipments sent by node $j$ amounts to

$$
o_{j}(t)=\sum_{i=1}^{N} \varphi_{j i}(t) q_{i}(t),
$$

where $N$ is the number of controlled nodes. Denoting by $M$ the number of sources and by $P=N+M$ the number of all the nodes in the network, the amount of goods received by node $i$ is determined as

$$
u_{i}(t)=\sum_{j=1}^{P} \varphi_{j i}\left(t-\tau_{j i}\right) q_{i}\left(t-\tau_{j i}\right) .
$$

Finally, taking into account all the node activities, the quantity of goods at controlled node $i$ (the on-hand stock) evolves according to

$$
x_{i}(t+1)=\left(x_{i}(t)+u_{i}(t)-d_{i}(t)\right)^{+}-o_{i}(t),
$$

where $d_{i}(t)$ is the market demand imposed on node $i$ in period $t .(f)^{+}=\max \{f, 0\}$ denotes the saturation function that makes the stock level nonnegative in the analyzed lost-sales system. The node interaction and principal system variables are illustrated in Figure 3.
2.3.3. Demand and Sales. In the assumed framework, the market demand imposed on the controlled nodes is modeled as an uncertain, bounded, time-varying function of time:

$$
0 \leq d_{i}(t) \leq d_{i}^{\max }
$$

where $d_{i}^{\max }$ denotes the highest value expected at node $i$ in the planning horizon. If $d_{i}(t)$ cannot be satisfied in full, the node effectuates the sales as much as the on-hand stock allows. Hence, the realized sales

$$
h_{i}(t)=\min \left\{x_{i}(t)+u_{i}(t), d_{i}(t)\right\} .
$$

As a result, using (9), the stock level evolution (7) might be expressed through

$$
x_{i}(t+1)=x_{i}(t)+u_{i}(t)-h_{i}(t)-o_{i}(t) .
$$

The effectiveness of goods distribution process in the considered network will be quantified through the customer satisfaction ratio (CS) defined as the percentage of realized sales with respect to the demands imposed on the controlled nodes. Denoting the total amount of goods requested within $T$ periods by $D$ and realized sales by $H$, one has

$$
\mathrm{CS}=\frac{H}{D}=\frac{\sum_{t=1}^{T} \sum_{i=1}^{N} h_{i}(t)}{\sum_{t=1}^{T} \sum_{i=1}^{N} d_{i}(t)} .
$$

2.4. State-Space Representation. For convenience of the analytical study and for efficient implementation on a computing machine, the model will be put to an appropriately chosen state-space form.

2.4.1. Topology Description. Let $\boldsymbol{\Phi}_{k}(t)$ denote a diagonal matrix corresponding to the goods traffic on the internode links with lead time $k \in\left\{1, \ldots, \tau_{\max }\right\}$ :

$$
\begin{array}{r}
\boldsymbol{\Phi}_{k}(t)=\operatorname{diag}\left\{\sum_{j: \tau_{j 1}=k} \varphi_{j 1}(t), \ldots, \sum_{j: \tau_{j N}=k} \varphi_{j N}(t)\right\}_{N \times N}, \\
j=1, \ldots, P .
\end{array}
$$

$\Phi_{0}(t)$ will be a hollow matrix corresponding to the internal transshipments sent in period $t$ :

$$
\boldsymbol{\Phi}_{0}(t)=-\left[\begin{array}{cccc}
0 & \varphi_{12}(t) & \cdots & \varphi_{1 N}(t) \\
\varphi_{21}(t) & 0 & \cdots & \varphi_{2 N}(t) \\
\vdots & \vdots & \ddots & \vdots \\
\varphi_{N 1}(t) & \varphi_{N 2}(t) & \cdots & 0
\end{array}\right] .
$$

$\varphi_{i i}(t)=0$ in (13) (the entries on the main diagonal) reflect the fact that no controlled node is a source of goods for itself. Moreover, since the network is directed, $\varphi_{j i}(t) \neq 0$ implies $\varphi_{i j}(t)=0$. An example matrix $\Phi_{0}$, corresponding to the network depicted in Figure 1, equals to 


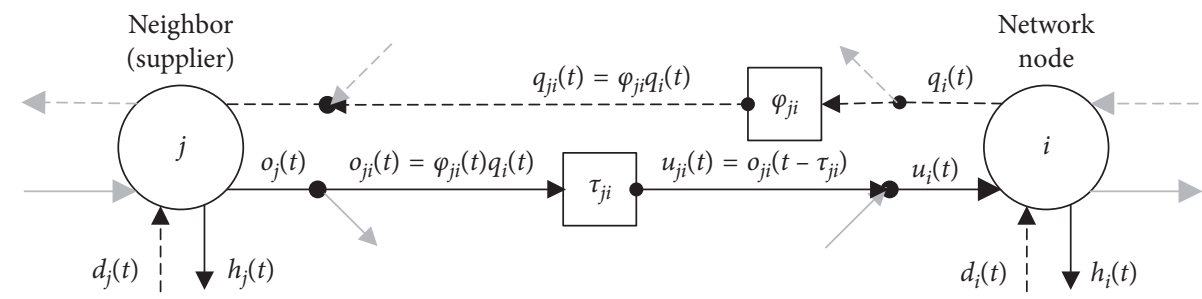

FIGURE 3: Main system variables: dashed lines represent the flow of information and solid lines represent the flow of goods. Aggregate flows are marked through thick lines.

$$
\Phi_{0}=-\left[\begin{array}{ccc}
0 & 0.4 & 0.3 \\
0 & 0 & 0.7 \\
0 & 0 & 0
\end{array}\right]
$$

and the other matrices

$$
\begin{aligned}
& \boldsymbol{\Phi}_{1}=\left[\begin{array}{lcc}
0 & 0 & 0 \\
0 & 0.4 & 0 \\
0 & 0 & 0
\end{array}\right], \\
& \boldsymbol{\Phi}_{2}=\left[\begin{array}{ccc}
1.0 & 0 & 0 \\
0 & 0 & 0 \\
0 & 0 & 0.7
\end{array}\right], \\
& \boldsymbol{\Phi}_{3}=\left[\begin{array}{lll}
0 & 0 & 0 \\
0 & 0 & 0 \\
0 & 0 & 0.3
\end{array}\right], \\
& \boldsymbol{\Phi}_{4}=\left[\begin{array}{lll}
0 & 0 & 0 \\
0 & 0.6 & 0 \\
0 & 0 & 0
\end{array}\right] .
\end{aligned}
$$

In the nominal operating conditions (no shortage), one can also define a matrix

$$
\boldsymbol{\Phi}=\sum_{k=0}^{\tau_{\max }} \boldsymbol{\Phi}_{k}
$$

with an important property from the perspective of further study specified in the following lemma.

Lemma 1. The inverse of $\Phi$ is a positive matrix.

Proof. It is required to show that the entries of $\Phi^{-1}$ are all nonnegative. First, it will be demonstrated that $\Phi$ is invertible.

When goods shortage does not occur, directly from the definition of system matrices (12) and (13), one has $\boldsymbol{\Phi}=\mathbf{I}+\boldsymbol{\Phi}_{0}$, where $\mathbf{I}$ is an $N \times N$ identity matrix. The entries of $\Phi_{0}$ are zero on the main diagonal, and they sum columnwise at most to -1 . Moreover, the unidirectional property of goods flow implies that whenever $\varphi_{i j} \neq 0$, its symmetric equivalent $\varphi_{j i}=0$. As a result, the calculation of the determinant and all the leading principal minors of $\Phi$ reduces to the triangular matrices (through the expansion formula) with ones on the main diagonal. Thus, the leading principal

minors and the determinant are all equal to one and, by virtue of Sylvester's criterion, $\boldsymbol{\Phi}$ is positive definite and has an inverse.

As $\left\|\Phi_{0}\right\|<1$, where $\|\cdot\|$ denotes induced matrix norm, $\Phi^{-1}$ can be written in the form of a series

$$
\boldsymbol{\Phi}^{-1}=\left(\mathbf{I}-\left(-\boldsymbol{\Phi}_{0}\right)\right)^{-1}=\mathbf{I}+\left(-\boldsymbol{\Phi}_{0}\right)+\left(-\boldsymbol{\Phi}_{0}\right)^{2}+\cdots .
$$

Since all the entries of $-\boldsymbol{\Phi}_{0}$ are nonnegative, according to (17), so are the entries of $\boldsymbol{\Phi}^{-1} \cdot \boldsymbol{\Phi}^{-1}$ is thus a positive matrix.

2.4.2. Dynamical Description. Having grouped the variables describing the node interaction into the vectors given in Table 1, the system dynamics may be described in the following state-space:

$$
\mathbf{x}(t+1)=\mathbf{x}(t)+\sum_{k=0}^{\tau_{\max }} \boldsymbol{\Phi}_{k}(t) \mathbf{q}(t-k)-\mathbf{h}(t) .
$$

With no input preceding the phase of goods distribution, i.e., $\mathbf{q}(t)=0$ for $t<0$, using (18), the stock level in any period $t>0$ may be determined using a nonrecurrent relation

$$
\begin{aligned}
\mathbf{x}(t)= & \mathbf{x}(0)+\sum_{k=0}^{\tau_{\max }} \sum_{l=0}^{t-1} \Phi_{k}(l-k) \mathbf{q}(l-k) \\
& -\sum_{l=0}^{t-1} \mathbf{h}(l)=\mathbf{x}(0)+\sum_{i=0}^{\tau_{\max }} \sum_{l=0}^{t-k-1} \boldsymbol{\Phi}_{k}(l) \mathbf{q}(l)-\sum_{l=0}^{t-1} \mathbf{h}(l) .
\end{aligned}
$$

2.5. Classical Base-Stock Policy. The base-stock policy is one of the classical inventory management strategies in logistics which determine the order quantity according to (demand prediction neglected)

$$
q_{i}(t)=x_{i}^{\mathrm{TIL}}-x_{i}(t)-\Delta_{i}(t)
$$

where $x_{i}^{\mathrm{TIL}}$ is the target inventory level and

$$
\Delta_{i}(t)=\sum_{j \in \Theta_{j}} \sum_{l=t-\tau_{j i}}^{t-1} \varphi_{j i}(l) q_{j i}(l),
$$

represents the goods in transit coming to node $i$.

Let $\mathbf{x}^{\mathrm{TIL}}=\left[x_{i}^{\mathrm{TIL}}, \ldots, x_{N}^{\mathrm{TIL}}\right]^{\prime}$ denote the vector of target inventory levels for all the controlled nodes. Using state-space 
description (12)-(18), the CBS policy for the analyzed goods distribution network can be represented in the vector form as

$$
\mathbf{q}(t)=\mathbf{x}^{\mathrm{TIL}}-\mathbf{x}(t)-\sum_{k=0}^{\tau_{\max }} \sum_{l=t-k}^{t-1} \boldsymbol{\Phi}_{k}(l) \mathbf{q}(l) .
$$

The CBS policy, when deployed in a distributed way to preserve the autonomy of ordering decisions at the network nodes (which can then be handled independently by different organizations), brings a risk of system underutilization, or loss of stability in the face of uncertain demand and delayed goods reflow. Two theorems given below demonstrate formally that this inopportune situation does not occur, irrespective of the demand pattern conforming to quite general conditions (8).

First, under the assumed initial conditions: $\mathbf{q}(t<0)=0$ and $\mathbf{q}(0)=\mathbf{x}^{\mathrm{TIL}}-\mathbf{x}(0)$. Since one is basically free in the choice of $\mathbf{x}^{\text {TIL }}$, it can be adjusted with respect to the initial resources $\mathbf{x}(0)$ so that a nonnegative order is placed by any node. In turn, for $t>0$, the ordering signal satisfies the condition specified in the following theorem.

Theorem 1. For any period $t>0$, the ordering signal generated using the CBS strategy is nonnegative and bounded.

Proof. By applying (19) into (22), the quantity of goods in the replenishment order established in period $t$ may be expressed as

$$
\mathbf{q}(t)=\mathbf{x}^{\mathrm{TIL}}-\mathbf{x}(0)-\sum_{k=0}^{\tau_{\max }} \sum_{l=0}^{t-1} \boldsymbol{\Phi}_{k}(l) \mathbf{q}(l)+\sum_{l=0}^{t-1} \mathbf{h}(l) .
$$

Consequently,

$$
\begin{aligned}
\mathbf{q}(t+1)= & \mathbf{x}^{\mathrm{TIL}}-\mathbf{x}(0)-\sum_{k=0}^{\tau_{\max }} \sum_{l=0}^{t} \boldsymbol{\Phi}_{k}(l) \mathbf{q}(l)+\sum_{l=0}^{t} \mathbf{h}(l) \\
= & \mathbf{x}^{\mathrm{TIL}}-\mathbf{x}(0)-\sum_{k=0}^{\tau_{\max }} \sum_{l=0}^{t-1} \boldsymbol{\Phi}_{k}(l) \mathbf{q}(l)+\sum_{l=0}^{t-1} \mathbf{h}(l) \\
& -\sum_{k=0}^{\tau_{\max }} \boldsymbol{\Phi}_{k}(t) \mathbf{q}(t)+\mathbf{h}(t) \\
= & \mathbf{q}(t)-\sum_{k=0}^{\tau_{\max }} \boldsymbol{\Phi}_{k}(t) \mathbf{q}(t)+\mathbf{h}(t) \\
= & {\left[\mathbf{I}-\sum_{k=0}^{\tau_{\max }} \boldsymbol{\Phi}_{k}(t)\right] \mathbf{q}(t)+\mathbf{h}(t) . }
\end{aligned}
$$

Since $\forall_{i} 0 \leq \sum_{j \in \Theta_{i}} \varphi_{j i}(t) \leq 1$, one has

$$
\begin{gathered}
\mathbf{I}-\sum_{k=0}^{\tau_{\max }} \boldsymbol{\Phi}_{k}(t)=\mathbf{I}-\sum_{k=1}^{\tau_{\max }} \boldsymbol{\Phi}_{k}(t)-\boldsymbol{\Phi}_{0}(t) \geq \mathbf{I}-\mathbf{I} \\
-\boldsymbol{\Phi}_{0}(t)=-\boldsymbol{\Phi}_{0}(t)>0 .
\end{gathered}
$$

The vector of realized sales $\mathbf{h}(t)$ is nonnegative (component wise). Thus, by applying (25) to (24), one obtains $\mathbf{q}(t+1) \geq 0$. On the other hand, $\left\|\mathbf{I}-\sum_{k=0}^{\tau_{\max }} \boldsymbol{\Phi}_{k}(t)\right\|<1$. Hence, since (24) represents the dynamics of an asymptotically stable linear system with state $\mathbf{q}(t)$ and bounded input $\mathbf{h}(t)$, $\mathbf{q}(t)$ is bounded.
Theorem 2. Under the CBS policy, the on-hand stock at the network nodes does not exceed the target level, i.e.,

$$
\underset{t \geq 0}{\forall} \mathbf{x}(t) \leq \mathbf{x}^{\mathrm{TIL}} \text {. }
$$

Proof. Initially, $\mathbf{x}(0) \leq \mathbf{x}^{\mathrm{TIL}}$. Assume that in a period $t>0$, $\mathbf{x}(t) \leq \mathbf{x}^{\text {TIL }}$. It will be demonstrated that using this assumption, the resources accumulated in the next period- $\mathbf{x}(t+1)$-do not exceed $\mathbf{x}^{\mathrm{TIL}}$.

Since the biggest stock increase occurs under no shortage $\left(\Phi_{k}(t)=\Phi_{k}\right)$, one has from (18)

$$
\mathbf{x}(t+1) \leq \mathbf{x}(t)+\sum_{k=0}^{\tau_{\max }} \Phi_{k} \mathbf{q}(t-k)-\mathbf{h}(t) .
$$

Using (23) in (27) yields

$$
\begin{aligned}
\mathbf{x}(t+1) \leq & \mathbf{x}(t)+\sum_{k=0}^{\tau_{\max }} \boldsymbol{\Phi}_{k}\left[\mathbf{x}^{\mathrm{TIL}}-\mathbf{x}(0)-\sum_{i=0}^{\tau_{\max }} \sum_{l=0}^{t-i-1} \boldsymbol{\Phi}_{i}(l) \mathbf{q}(l)\right. \\
& \left.+\sum_{l=0}^{t-i-1} \mathbf{h}(l)\right]-\mathbf{h}(t) \\
= & \mathbf{x}(t)+\sum_{k=0}^{\tau_{\max }} \boldsymbol{\Phi}_{k} \mathbf{x}^{\mathrm{TIL}}-\sum_{k=0}^{\tau_{\max }} \boldsymbol{\Phi}_{k}\left[\mathbf{x}(0)+\sum_{i=0}^{\tau_{\max }} \sum_{l=0}^{t-i-1} \boldsymbol{\Phi}_{i}(l) \mathbf{q}(l)\right. \\
& \left.-\sum_{l=0}^{t-1} \mathbf{h}(l)\right]-\sum_{k=0}^{\tau_{\max }} \boldsymbol{\Phi}_{k} \sum_{l=t-k}^{t-1} \mathbf{h}(l)-\mathbf{h}(t) .
\end{aligned}
$$

The terms inside the square brackets in (28) actually equal $\mathbf{x}(t)$. Hence, by applying the definition $\boldsymbol{\Phi}=\sum_{k=0}^{\tau_{\max }} \boldsymbol{\Phi}_{k}$, one arrives at

$$
\mathbf{x}(t+1) \leq \mathbf{x}(t)+\boldsymbol{\Phi} \mathbf{x}^{\mathrm{TIL}}-\boldsymbol{\Phi} \mathbf{x}(t)-\sum_{k=0}^{\tau_{\max }} \boldsymbol{\Phi}_{k} \sum_{l=t-k}^{t-1} \mathbf{h}(l)-\mathbf{h}(t) .
$$

As $\boldsymbol{\Phi}=\mathbf{I}+\boldsymbol{\Phi}_{0}$ (see the proof of Lemma 1$), \mathbf{x}(t)-\boldsymbol{\Phi}$ $\mathbf{x}(t)=-\boldsymbol{\Phi}_{0} \mathbf{x}(t)$ and

$$
\mathbf{x}(t+1) \leq \mathbf{x}^{\mathrm{TIL}}+\boldsymbol{\Phi}_{0}\left[\mathbf{x}^{\mathrm{TIL}}-\mathbf{x}(t)\right]-\sum_{k=0}^{\tau_{\max }} \boldsymbol{\Phi}_{k} \sum_{l=t-k}^{t-1} \mathbf{h}(l)-\mathbf{h}(t) .
$$

By definition, $\Phi_{0}$ has all the entries negative or null. Since $\mathbf{h}$ has only nonnegative components, by virtue of the mathematical induction one may conclude that $\mathbf{x}(t+1) \leq$ $\mathbf{x}^{\text {TIL }}$.

Theorem 1 shows that even though implemented without any knowledge about the network structure, the CBS policy generates a feasible sequence of ordering decisions. Theorem 2 , in turn, proves that the stock level never exceeds the target value, irrespective of the actually realized sales. What remains to discuss is how to adjust $\mathbf{x}^{\text {TIL }}$ so that cost-efficient system operation is ensured.

Normally, one needs to recur to the numerical simulations for a given demand pattern to see how the choice of target level influences the costs. In order to limit the considerable search space, a minimum value that provides full 
customer satisfaction under any demand conforming to (8) can be indicated. A steady-state ordering sequence $\mathbf{q}_{\mathrm{ss}}$ in response to steady-state demand $\mathbf{d}_{\mathrm{ss}}$ may be determined from (24) as $\mathbf{q}_{\mathrm{ss}}=\mathbf{q}_{\mathrm{ss}}-\sum_{k=0}^{\tau_{\max }} \boldsymbol{\Phi}_{k}^{\mathrm{ss}} \mathbf{q}_{\mathrm{ss}}+\mathbf{d}_{\mathrm{ss}} \Longrightarrow \mathbf{q}_{\mathrm{ss}}=\left(\sum_{k=0}^{\tau_{\max }}\right.$ $\left.\boldsymbol{\Phi}_{k}^{s s}\right)^{-1} \mathbf{d}_{s s}$, where $\boldsymbol{\Phi}_{k}^{\text {ss }}$ represents $\boldsymbol{\Phi}_{k}(t)$ in the steady state. Then, using (22), the steady-state stock level becomes

$$
\begin{aligned}
\mathbf{x}_{\mathrm{ss}}= & \mathbf{x}^{\mathrm{TIL}}-\mathbf{q}_{\mathrm{ss}}-\sum_{k=1}^{\tau_{\max }} k \boldsymbol{\Phi}_{k}^{s s} \mathbf{q}_{\mathrm{ss}}=\mathbf{x}^{\mathrm{TIL}} \\
& -\left(\mathbf{I}+\sum_{k=1}^{\tau_{\max }} k \boldsymbol{\Phi}_{k}^{\mathrm{ss}}\right)\left(\sum_{k=0}^{\tau_{\max }} \boldsymbol{\Phi}_{k}^{\mathrm{ss}}\right)^{-1} \mathbf{d}_{\mathrm{ss}} .
\end{aligned}
$$

In the extreme case, $\mathbf{d}_{\mathrm{ss}}=\mathbf{d}_{\max }$ and $\boldsymbol{\Phi}_{k}^{\mathrm{ss}}=\boldsymbol{\Phi}_{k}$. However, setting $\mathbf{x}^{\text {TIL }}$ so that expression (31) is positive results in a positive stock level during the goods redistribution, and full customer satisfaction is achieved. The lowest value of $\mathbf{x}^{\text {TIL }}$ when it happens,

$$
\mathbf{x}_{\text {init }}^{\mathrm{TIL}}=\left(\mathbf{I}+\sum_{k=1}^{\tau_{\max }} k \boldsymbol{\Phi}_{k}\right) \boldsymbol{\Phi}^{-1} \mathbf{d}_{\max },
$$

will be used as a departure point in the numerical procedures to initiate genetic-algorithm-based search for optimum cost.

2.6. Networked Base-Stock Strategy. The CBS policy takes a local view of the logistic system, i.e., the decisions are made solely based on the interaction with directly connected neighbors. The idea behind a new (NBS) policy is to judiciously introduce the information about network interconnection structure so that the stocks (and as a consequence the holding costs) are reduced while maintaining a similar level of customer satisfaction as the CBS one.

According to the NBS policy, the quantity of goods to be acquired to replenish the stock by the controlled nodes will be calculated as

$$
\mathbf{q}(t)=\boldsymbol{\Phi}^{-1}\left[\mathbf{x}^{\mathrm{TIL}}-\mathbf{x}(t)-\sum_{k=0}^{\tau_{\max }} \sum_{l=t-k}^{t-1} \boldsymbol{\Phi}_{k}(l) \mathbf{q}(l)\right] .
$$

Comparing with (22), one may notice a similar calculation scheme, involving the record of on-hand stock and goods in transit, as in the CBS policy. Therefore, the implementation complexity does not increase. What differs is a matrix scaling factor, $\Phi^{-1}$, that is used for a more exact adjustment of ordering decisions in the networked structure, as discussed in a latter part of this section.

Directly from (33), under the assumed zero initial input, one has $\mathbf{q}(0)=\Phi^{-1}\left[\mathbf{x}^{\mathrm{TIL}}-\mathbf{x}(0)\right]$, which is upper-bounded and nonnegative if one assigns $\mathbf{x}^{\mathrm{TIL}} \geq \mathbf{x}(0)$. The theorem below shows that this property holds true for any $t>0$.

Theorem 3. For any period $t>0$, the ordering signal generated using the NBS strategy is nonnegative and bounded.
Proof. Similarly as in (24), substituting (19) into (33) yields

$$
\begin{aligned}
\mathbf{q}(t+1)= & \boldsymbol{\Phi}^{-1}\left[\mathbf{x}^{\mathrm{TIL}}-\mathbf{x}(0)-\sum_{k=0}^{\tau_{\max }} \sum_{l=0}^{t} \boldsymbol{\Phi}_{k}(l) \mathbf{q}(l)+\sum_{l=0}^{t} \mathbf{h}(l)\right] \\
= & \boldsymbol{\Phi}^{-1}\left[\mathbf{x}^{\mathrm{TIL}}-\mathbf{x}(0)-\sum_{k=0}^{\tau_{\max }} \sum_{l=0}^{t-1} \boldsymbol{\Phi}_{k}(l) \mathbf{q}(l)+\sum_{l=0}^{t-1} \mathbf{h}(l)\right] \\
& -\boldsymbol{\Phi}^{-1}\left[\sum_{k=0}^{\tau_{\max }} \boldsymbol{\Phi}_{k}(t) \mathbf{q}(t)-\mathbf{h}(t)\right] \\
= & \mathbf{q}(t)-\boldsymbol{\Phi}^{-1} \sum_{k=0}^{\tau_{\max }} \boldsymbol{\Phi}_{k}(t) \mathbf{q}(t)+\boldsymbol{\Phi}^{-1} \mathbf{h}(t) \\
= & {\left[\mathbf{I}-\boldsymbol{\Phi}^{-1} \sum_{k=0}^{\tau_{\max }} \boldsymbol{\Phi}_{k}(t)\right] \mathbf{q}(t)+\boldsymbol{\Phi}^{-1} \mathbf{h}(t) . }
\end{aligned}
$$

Since $\left\|\Phi^{-1} \sum_{k=0}^{\tau_{\max }} \Phi_{k}(t)\right\| \leq 1$, all the eigenvalues of $\mathbf{I}-\boldsymbol{\Phi}^{-1} \sum_{k=0}^{\tau_{\max }} \boldsymbol{\Phi}_{k}(t)$ lie within the unit circle and $\mathbf{q}$, evolving according to (34), is bounded. Moreover, since $\mathbf{q}(0) \geq 0$ and $\mathbf{h}(t) \geq 0, \mathbf{q}(t) \geq 0$.

Corollary 1. Under no goods shortage, for any $t>0$, the ordering signal generated using the NBS strategy

$$
\mathbf{q}(t)=\Phi^{-1} \mathbf{d}(t-1) .
$$

Proof. When there is no shortage of resources to sustain the goods reflow in the network, $\mathbf{h}(t)=\mathbf{d}(t)$ and $\sum_{k=0}^{\tau_{\max }} \boldsymbol{\Phi}_{k}(t)=\boldsymbol{\Phi}$. Using this fact in the last line in (34) yields

$$
\begin{aligned}
\mathbf{q}(t) & =\left[\mathbf{I}-\boldsymbol{\Phi}^{-1} \boldsymbol{\Phi}\right] \mathbf{q}(t-1)+\boldsymbol{\Phi}^{-1} \mathbf{h}(t-1)=\boldsymbol{\Phi}^{-1} \mathbf{h}(t-1) \\
& =\boldsymbol{\Phi}^{-1} \mathbf{d}(t-1)
\end{aligned}
$$

Remark 1. In the traditionally considered framework of only the local interactions considered (and constant order-up-to levels), it can be shown that the CBS policy satisfies the condition $q_{i}(t)=d_{i}(t-1)$ [54], i.e., it repeats the last observed demand. It follows from (24) that this desirable, memoryless property no longer holds for the network constructs. Even for the nominal-no-shortage-operational conditions, one has $\mathbf{q}(t)=(\mathbf{I}-\boldsymbol{\Phi}) \mathbf{q}(t-1)+\mathbf{d}(t-1)$, which implicates past ordering decisions in the current one. Contrarily, relation (35) shows that the NBS policy keeps generation of the ordering sequence memoryless, thus avoiding the propagation of demand fluctuations in time and the unwanted spiral of events related, in principle, to the bullwhip effect. However, a more profound study of these phenomena from the perspective of complex networked structures is in place for future research work.

Theorem 4. Under the NBS policy the on-hand stock at the network nodes does not exceed the target level. 
Proof. Similarly as in the proof of Theorem 2, one has $\mathbf{x}(0) \leq$ $\mathbf{x}^{\text {TIL }}$. The biggest stock increase occurs under no shortage. Using (23) in (27) with $\boldsymbol{\Phi}_{k}(t)=\boldsymbol{\Phi}_{k}$ yields

$$
\begin{aligned}
\mathbf{x}(t+1) \leq & \mathbf{x}(t)+\sum_{k=0}^{\tau_{\max }} \boldsymbol{\Phi}_{k} \boldsymbol{\Phi}^{-1} \mathbf{x}^{\mathrm{TIL}}-\sum_{k=0}^{\tau_{\max }} \boldsymbol{\Phi}_{k} \boldsymbol{\Phi}^{-1}[\mathbf{x}(0) \\
& \left.+\sum_{i=0}^{\tau_{\max }} \sum_{l=0}^{t-i-1} \boldsymbol{\Phi}_{i}(l) \mathbf{q}(l)-\sum_{l=0}^{t-1} \mathbf{h}(l)\right] \\
& -\sum_{k=0}^{\tau_{\max }} \boldsymbol{\Phi}_{k} \boldsymbol{\Phi}^{-1} \sum_{l=t-k}^{t-1} \mathbf{h}(l)-\mathbf{h}(t) .
\end{aligned}
$$

Using (19) and (16) in (37), one obtains

$$
\begin{gathered}
\mathbf{x}(t+1) \leq \mathbf{x}(t)+\Phi \Phi^{-1} \mathbf{x}^{\mathrm{TIL}}-\boldsymbol{\Phi} \boldsymbol{\Phi}^{-1} \mathbf{x}(t)-\sum_{k=0}^{\tau_{\max }} \boldsymbol{\Phi}_{k} \boldsymbol{\Phi}^{-1} \sum_{l=t-k}^{t-1} \mathbf{h}(l) \\
-\mathbf{h}(t)=\mathbf{x}^{\mathrm{TIL}}-\sum_{k=0}^{\tau_{\max }} \boldsymbol{\Phi}_{k} \boldsymbol{\Phi}^{-1} \sum_{l=t-k}^{t-1} \mathbf{h}(l)-\mathbf{h}(t) .
\end{gathered}
$$

Since $\mathbf{h}(t) \geq 0$ in any period $t, \mathbf{x}(t+1) \leq \mathbf{x}^{\mathrm{TIL}}$.

It has been demonstrated formally in Theorems 2 and 4 that both the classical and networked policy limit the goods accumulation at the network nodes up to the target level, no matter the demand profile. Therefore, $\mathbf{x}^{\text {TIL }}$ indicates the warehouse space one needs to assign at the nodes to avoid emergency storage. Similar to the analysis of the CBS policy, one may establish a steady-state relationship between the imposed demand and the stock level. Considering (38), one has

$$
\mathbf{x}_{\mathrm{ss}}=\mathbf{x}^{\mathrm{TIL}}-\sum_{k=1}^{\tau_{\max }} k \boldsymbol{\Phi}_{k}^{\mathrm{ss}} \boldsymbol{\Phi}^{-1} \mathbf{d}_{\mathrm{ss}}-\mathbf{d}_{\mathrm{ss}} .
$$

Hence,

$$
\mathbf{x}_{\text {init }}^{\mathrm{TIL}}=\left(\mathbf{I}+\sum_{k=1}^{\tau_{\max }} k \boldsymbol{\Phi}_{k} \boldsymbol{\Phi}^{-1}\right) \mathbf{d}_{\max }
$$

provides a lower bound for the target level to reach the noshortage conditions in the networked system, for the maximum demand imposed throughout the entire distribution cycle. Comparing (32) with (40), one obtains the difference $\left(\boldsymbol{\Phi}^{-1}-\mathbf{I}\right) \mathbf{d}_{\max }$ in terms of safety stock in favor of the NBS policy. As documented in the numerical section for different, time-varying demands, the NBS policy will generally require a smaller safety stock to ensure a given service level than the CBS strategy, giving the holding cost advantage.

\section{Numerical Study}

The performance of resource management strategies has been investigated in a wide variety of numerical tests for the networks with different sizes, complexity, and operational conditions. In order to automate the tests, a dedicated computational engine has been created. Each test case proceeds as follows:
(1) Using the input parameters, number of controlled nodes $N$, number of external sources $M$, number of contact points with the market, and connection complexity $C$ quantified through the number of node suppliers, the network interconnection structure is constructed.

(2) Next, the simulation environment is prepared. It involves choosing the simulation time, type of demand distribution, and control policy (either CBS or NBS one).

(3) The optimization process is conducted, i.e., using continuous genetic algorithm (CGA) (implementation details given by Ignaciuk and Wieczorek [55]), an optimal $\mathbf{x}^{\text {TIL }}$ allocation is determined. The applied fitness function balances the priority of holding cost (HC) reduction vs. achieved customer satisfaction (11) through Fitness $=\left(1-\mathrm{HC} / \mathrm{HC}_{\text {initial }}\right)^{\alpha} \mathrm{CS}^{\beta}$, where $\alpha>0$ and $\beta>0$ are tuning parameters.

Altogether, $10^{7}$ test instances have been run. A few representative cases have been selected for closer examination in a latter part of this section with three major aspects in mind:

(1) Network size (Section 3.1)-3 interconnection structures encompassing $M=5$ sources and a varying number of controlled nodes: 5,10 , and 15 nodes

(2) Market impact (Section 3.2) - the network topology comprising $N=10$ controlled nodes and $M=5$ sources with the external demand imposed on a varying number of nodes: 3, 6, and 10 nodes

(3) Topological complexity (Section 3.3)-3 networks, each comprising 20 nodes $(N=15, M=5)$ and a different number of suppliers supporting each controlled node: 2, 4, and 6 suppliers, respectively

The other simulation parameters are set as follows:

(i) Planning horizon: $T=50$ periods

(ii) Lead-time delay: assigned randomly from the interval $[1,5]$

(iii) Market demand: generated according to the Gamma distribution with shape and scale coefficients equal to 5 and 10 , respectively

(iv) CGA: 10 individuals in a population, $2 \%$ mutation probability, stop condition ( $10^{4}$ generations evaluated), or $3 \cdot 10^{3}$ generations without improvement

3.1. Network Size. The first group of tests aims at assessing the system performance, in particular $\mathrm{HC}$, in relation to the number of nodes subject to inventory management decisions. All the examined topologies assume a fixed number of sources $(M=5)$ and a growing number of controlled nodes $N$, as illustrated in Figure 4. The external demands are imposed on every node throughout the entire simulation interval $[0, T]$. The computational complexity increases according to $\lceil N / 3\rceil$. The optimization procedure, involving 


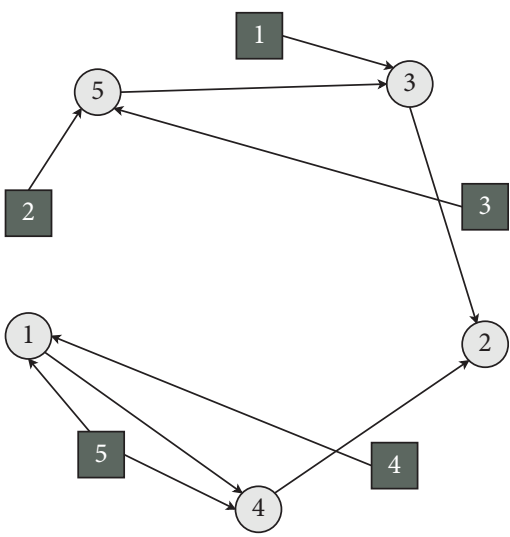

(a)

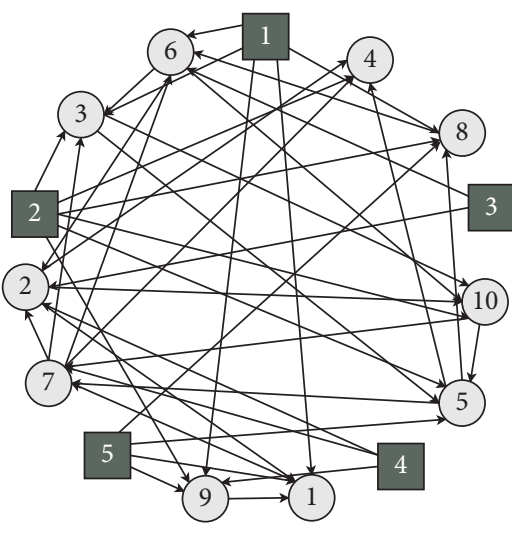

(b)

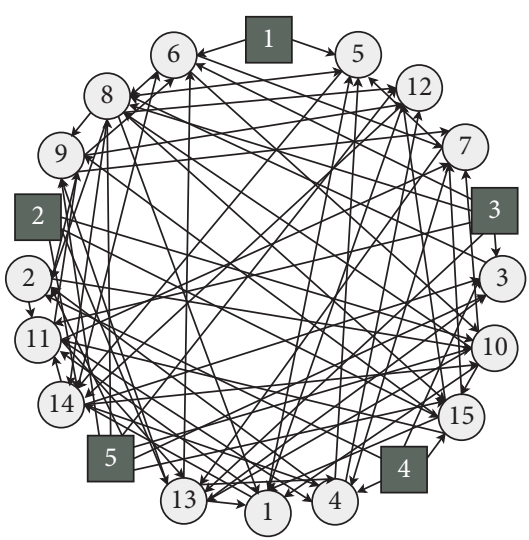

(c)

FIGURE 4: Interconnection structures with a growing number of controlled nodes: (a) 5, (b) 10, and (c) 15 nodes.

CGA parametrized via $\alpha=2$ and $\beta=15$, is assumed to provide full customer satisfaction with minimum $\mathrm{HC}$.

The data gathered in Table 2 (and illustrated graphically in Figure 5) indicate that the bigger the number of controlled nodes, the higher the savings obtained through the application of NBS strategy instead of the CBS one. In the case of simple network topology from Figure 4(a), HC is reduced from $2.02 \cdot 10^{4}$ to $1.83 \cdot 10^{4}$ units, i.e., by $9 \%$. For the network with 10 controlled nodes (b), the reduction amounts to $20 \%$, and for the largest network from Figure 4(c), the application of the NBS policy enables one to bring $\mathrm{HC}$ down by $26 \%$ with respect to the CBS strategy.

3.2. Impact of Local Market. Another significant aspect of logistic network performance relates to the interaction with external market, i.e., the number of contact points (or the number of controlled nodes affected directly by external demand). In the tests reported in this section, an interconnection structure comprising 20 nodes $(N=15$ controlled nodes and $M=5$ sources) depicted in Figure 6 has been selected. The number of contact points (marked in bold in Figure 6) equals to 5, 10, and 15, for cases 6(a)-6(c), respectively.

The results are summarized in Table 3 . The first network under consideration (a) assumes that the market demand is imposed on $30 \%$ of the controlled nodes. The resource distribution according to the CBS policy generated $\mathrm{HC}=1.47 \cdot 10^{4}$ units and $1.14 \cdot 10^{4}$ units in the case of NBS policy. The advantage in favor of the NBS policy thus amounts to $23 \%$. With the number of contact points increased twice (in the same topology), the $\mathrm{HC}$ reduction $\approx 21 \%$. Finally, when each controlled node serves as a contact point answering the external demand, the cost reduction owing to the application of NBS instead of CBS policy increases up to $27 \%$ (which is consistent with the cases considered in Section 3.1). The obtained data show that NBS strategy outperforms the classical one irrespective of the number of contact points, yet the actual benefit does not necessarily follow an increasing trend.
TABLE 2: The impact of network size on the performance of CBS and NBS policies.

\begin{tabular}{lccc}
\hline \multirow{2}{*}{$N$} & \multicolumn{2}{c}{ HC (units) } & Savings (\%) \\
\hline 5 & CBS & NBS & 9 \\
10 & 20,245 & 18,349 & 20 \\
15 & 31,121 & 24,816 & 26 \\
\hline
\end{tabular}

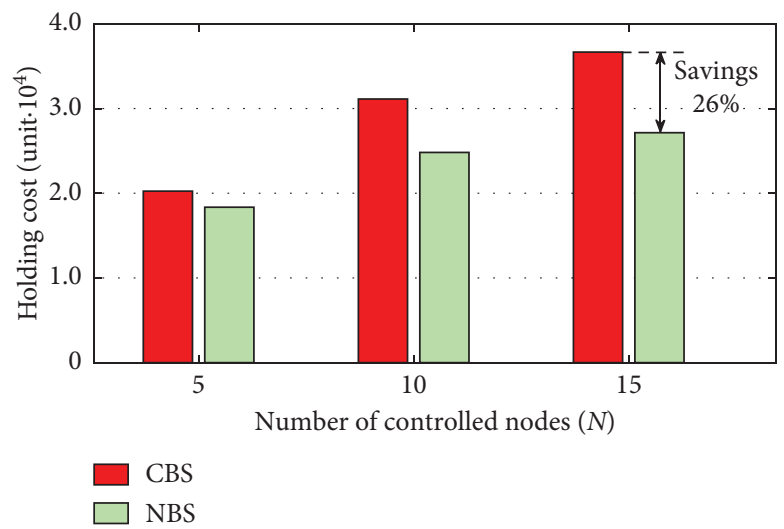

Figure 5: Holding cost vs. number of controlled nodes.

3.3. Topological Complexity. In order to assess the influence of topological complexity, three distribution systems are investigated:

(i) Weakly connected network $\mathrm{N} 1(N=15, M=5, C=2)$

(ii) Medium complexity network $\mathrm{N} 2(N=15, M=5, C=4)$

(iii) Strongly connected network $\mathrm{N} 3(N=15, M=5, C=6)$

Recall that $C$ denotes the number of node suppliers. The interconnection structures are sketched in Figure 7. For network N1 illustrated in Figure 7(a), the initial $\mathbf{x}^{\mathrm{TIL}}$ vectors, calculated according to (32) for the CBS policy and according to (40) for the NBS one, lead to HC of $6.6 \cdot 10^{5}$ units. With the granularity of one unit, there are $7.8 \cdot 10^{45}$ potential solutions in the search space for the brute-force optimization approach. Hence, even for a low-complexity 


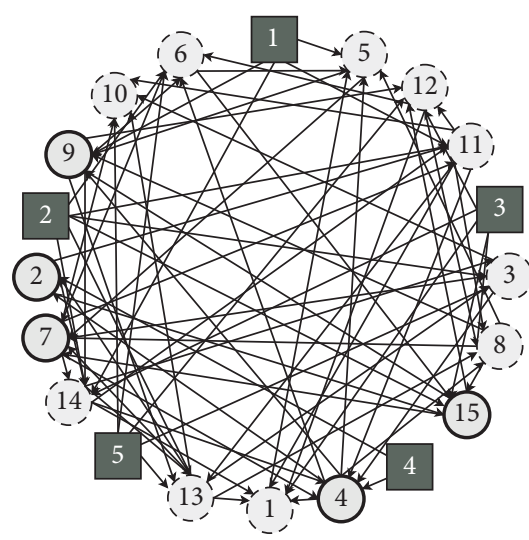

(a)

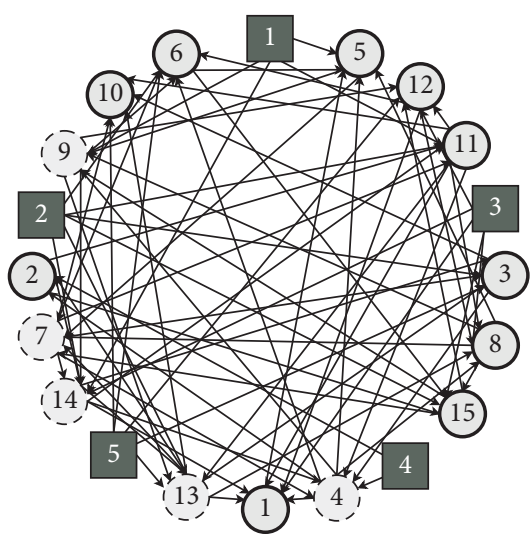

(b)

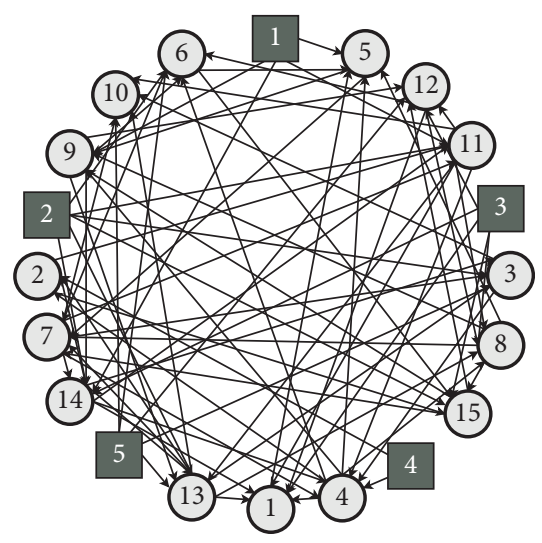

(c)

FIGURE 6: Interconnection structures with growing impact of local market: (a) 5, (b) 10, and (c) 15 contact points.

TABLE 3: The impact of market impact on the performance of CBS and NBS policies.

\begin{tabular}{lccc}
\hline Number of contact points & HC (units) & NBS & Savings (\%) \\
\hline 5 & CBS & 11,382 & 23 \\
10 & 14,733 & 35,881 & 21 \\
15 & 45,450 & 34,295 & 27 \\
\hline
\end{tabular}

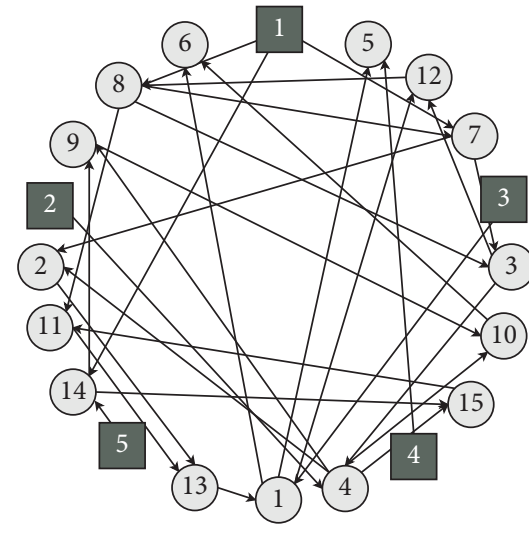

(a)

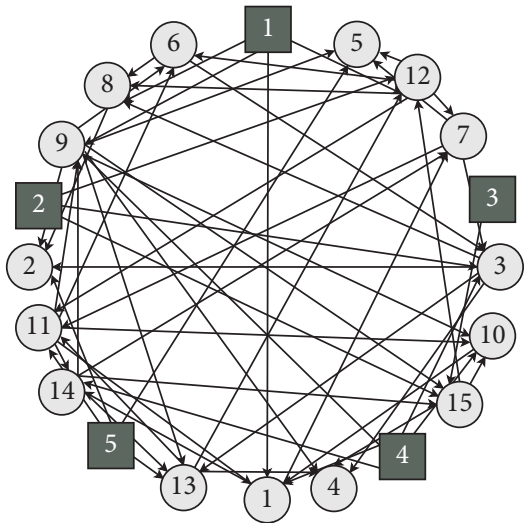

(b)

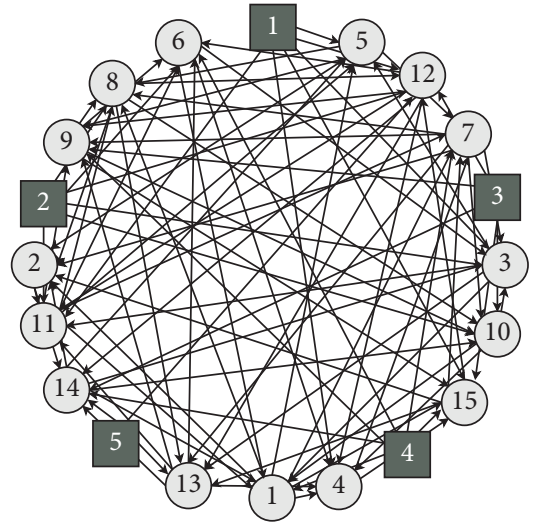

(c)

FIGURE 7: Interconnection structures with growing topological complexity: (a) 2, (b) 4, and (c) 6 node suppliers.

network, one needs to recur to approximate solutions, here CGA.

The charts in Figure 8 depict $\mathbf{x}^{\mathrm{TIL}}$ obtained for full customer satisfaction. For a majority of controlled nodes, the determined target level is smaller for the NBS policy than for the classical one. In the case of network N1, this difference translates to $33 \%$ cost reduction in favor of the networked policy $\left(5.2 \cdot 10^{4}\right.$ units for the CBS policy vs. $3.5 \cdot 10^{4}$ units for the NBS one). The plots in Figures $8(\mathrm{~b})$ and $8(\mathrm{c})$ reveal similar tendency for networks N2 and N3.

Figure 9 illustrates how the holding cost changes in relation to different levels of intended customer satisfaction. The profitability in applying the NBS policy rises with the increase in the desired satisfaction level. While for low service levels, e.g., 50\%, the costs generated by both policies are similar, in the typical settings of CS $>70 \%$, the networked policy generates significantly smaller holding costs. Comparing the plots from Figures 9(a)-9(c), one can notice a positive impact of enlarged internode linkage on decreasing the holding costs for a given service level. The benefits are more substantial in the case of networked policy, which generates smaller costs in all the investigated scenarios. This observation is confirmed by the data grouped in Table 4, which show that the savings (CBS-CNS)/CBS for a given topology and the same demand imposed on the controlled nodes can reach the level of $60 \%$. In the table, the cases of achieving full customer satisfaction (CS $=100 \%)$ are marked in bold. 


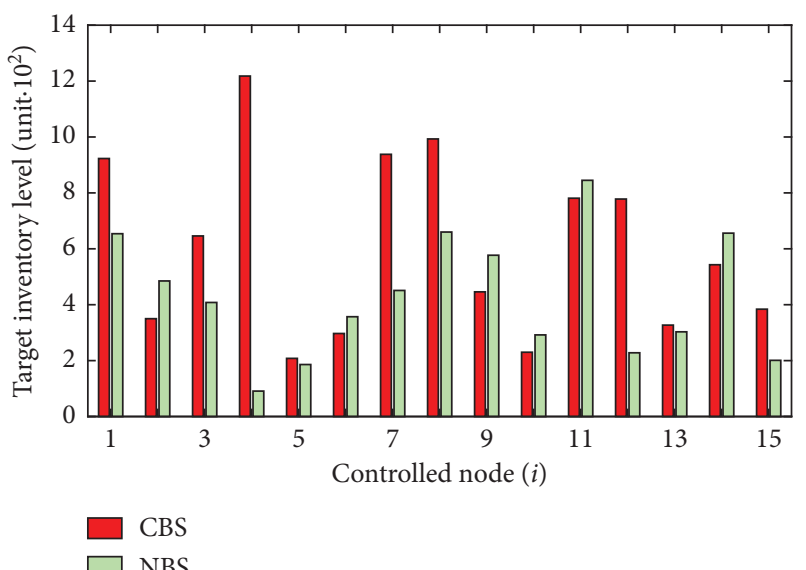

(a)

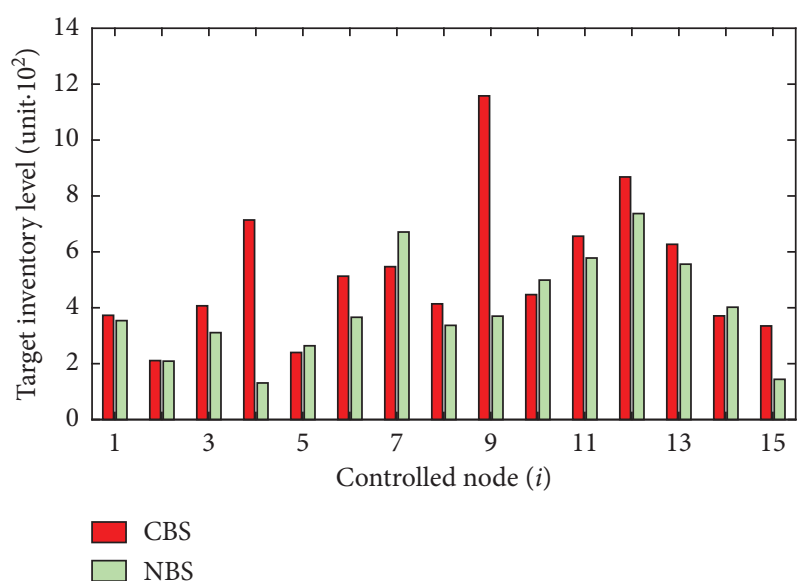

(b)

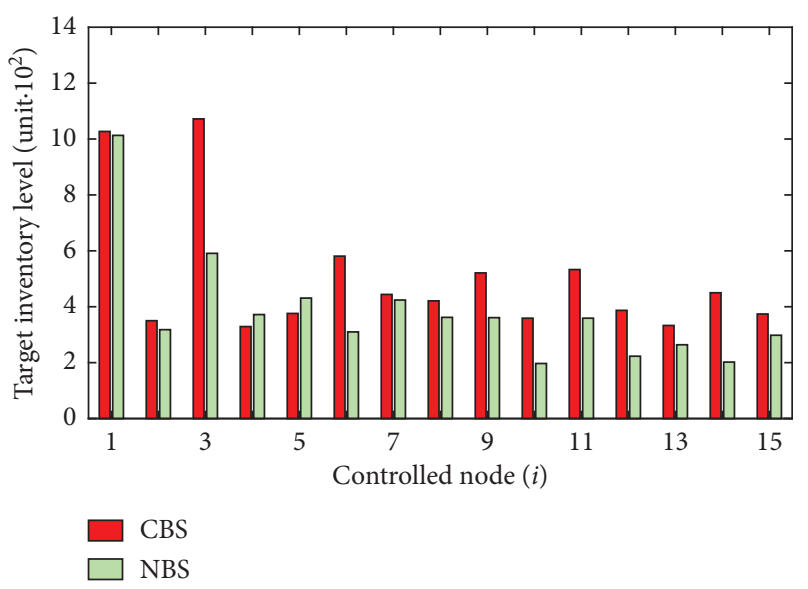

(c)

FIgURE 8: $\mathbf{x}^{\text {TIL }}$ for CBS and NBS policies with growing topological complexity: (a) 2, (b) 4, and (c) 6 node suppliers.

The conducted tests show a clear benefit of employing the network structural information into the ordering decision scheme. For network N2, the optimal cost equals $3.8 \cdot 10^{4}$ units (CBS) and $2.1 \cdot 10^{4}$ units (NBS), respectively. The reduction of holding cost is more profound than in the case of network $\mathrm{N} 1$ for all the customer satisfaction levels. In network $\mathrm{N} 2$, similarly to network $\mathrm{N} 1$, the higher the intended CS, the bigger the benefits from using the NBS strategy. These observations are confirmed for the strongly connected network N3.

\section{Summary and Conclusions}

This paper examines the use of base-stock inventory policies in goods distribution networks with nontrivial interconnection structures. The nodes form links with other nodes in the system in an arbitrary way. The goods redistribution process is subject to positive lead-time delay, potentially different at each internode link, and uncertain demand may be placed at any controlled node. The paper evaluates analytically the classical base-stock policy under demand uncertainty in a robust control framework. It is formally shown that despite the delay in goods transshipment, the CBS policy always generates nonnegative (thus feasible) ordering signal and finite, precisely determined stock level for any demand pattern from a given interval. Only the upper estimate of demand is required. Next, using the implications from the classical policy behavior, a new base-stock policy is established. This networked policy, by incorporating basic topological information, is shown to generate smaller holding costs while maintaining all the essential properties of the classical one.

As evaluated in extensive numerical tests, involving genetic algorithms for policy parameter tuning, the networked policy generates smaller costs than the classical one. Three aspects of system performance have been given emphasis: network size (quantified through the number of nodes subject to ordering decisions), relations with external actors (quantified through the number of contact points), and complexity (quantified through the linkage density). Depending on the service-level setting, the benefits may range from $9 \%$ to as much as $60 \%$. The cost savings have been observed in all the test cases. With full customer satisfaction as a target, the cost savings increase from 9\%, through $21 \%$, up to $27 \%$ with the network size growing from 5 , through 10, up to 15 controlled nodes. A similar trend, though not strictly increasing, is observed for varying the number of nodes on which the external demand is imposed. 


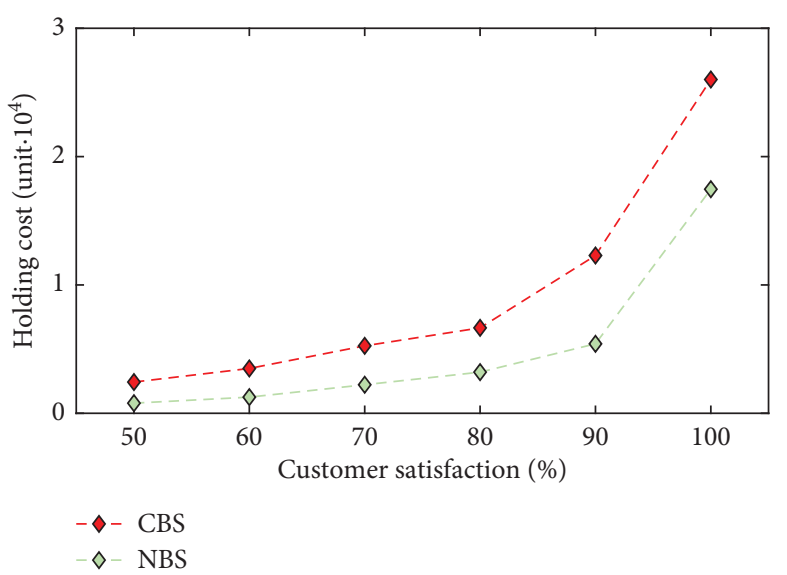

(a)

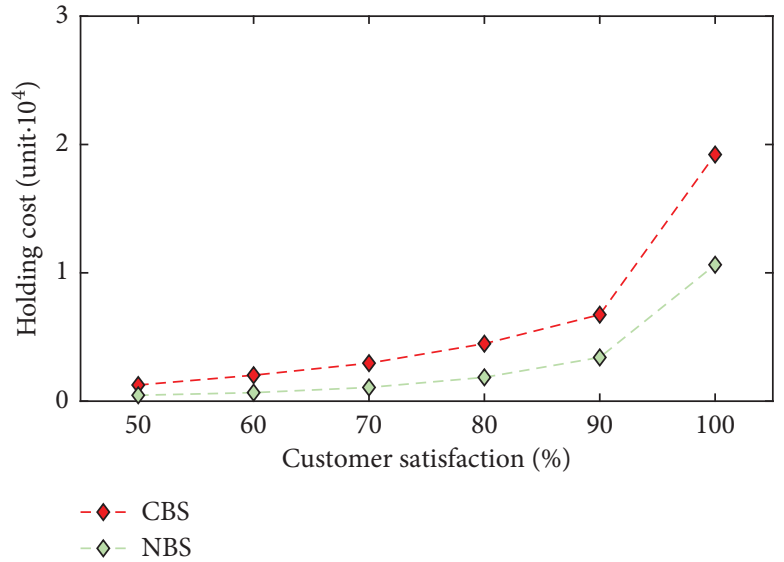

(b)

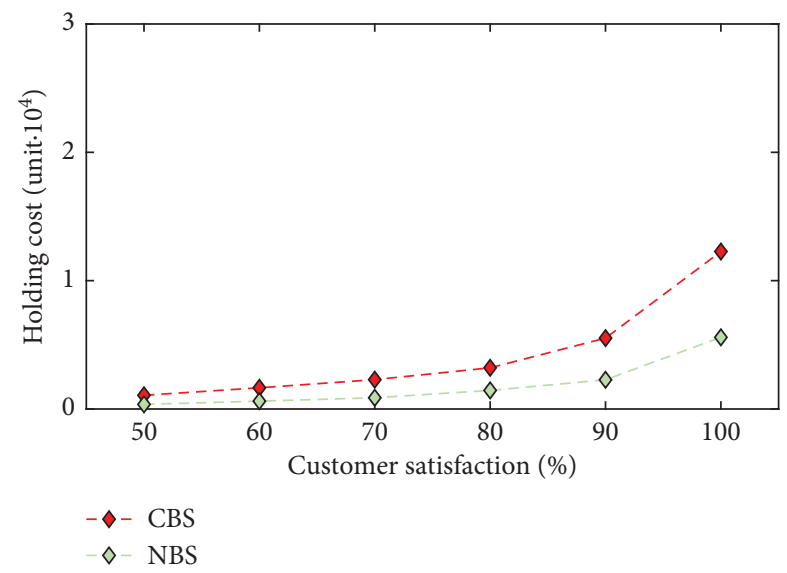

(c)

Figure 9: Holding cost vs. customer satisfaction for networks: (a) N1, (b) N2, and (c) N3.

TABLE 4: Performance of CBS and NBS policies.

\begin{tabular}{|c|c|c|c|c|}
\hline \multirow{2}{*}{ Network } & \multirow{2}{*}{ Desired CS (\%) } & \multicolumn{2}{|c|}{ HC (units) } & \multirow{2}{*}{ Savings (\%) } \\
\hline & & CBS & NBS & \\
\hline N1 & 50 & 4,867 & 1,577 & 68 \\
\hline N1 & 60 & 6,985 & 2,517 & 64 \\
\hline N1 & 70 & 10,510 & 4,447 & 58 \\
\hline N1 & 80 & 13,312 & 6,412 & 52 \\
\hline N1 & 90 & 24,587 & 10,824 & 56 \\
\hline N1 & 100 & 52,014 & 34,918 & 33 \\
\hline N2 & 50 & 2,518 & 920 & 63 \\
\hline N2 & 60 & 4,047 & 1,329 & 67 \\
\hline N2 & 70 & 5,923 & 2,140 & 64 \\
\hline N2 & 80 & 8,959 & 3,714 & 59 \\
\hline N2 & 90 & 13,481 & 6,817 & 49 \\
\hline N2 & 100 & 38,434 & 21,259 & 45 \\
\hline N3 & 50 & 2,137 & 721 & 66 \\
\hline N3 & 60 & 3,305 & 1,224 & 63 \\
\hline N3 & 70 & 4,574 & 1,748 & 62 \\
\hline N3 & 80 & 6,420 & 2,899 & 55 \\
\hline N3 & 90 & 11,048 & 4,551 & 59 \\
\hline N3 & 100 & 24,538 & 11,169 & 54 \\
\hline
\end{tabular}


The biggest advantage of using the networked policy has been observed in the tests measuring the impact of topological complexity (Section 3.3). When full customer satisfaction (under minimum holding costs) is to be obtained, the savings can reach $60 \%$.

While introducing minor computational change (the value scaled by a constant matrix), the networked policy provides substantial economic advantage over the classical order-up-to policy. However, it assumes centralized operation under the auspices of an organization sharing common topological information. By contrast, the classical policy can be deployed in a distributed way, independently at each node. Therefore, the networked policy is recommended for organizations with well-defined, reliable information channels. The influence of information distortion requires deeper evaluation as a subject of further study.

\section{Data Availability}

The numerical data related to the presented results can be made available from the corresponding author upon request.

\section{Conflicts of Interest}

The authors declare that they have no conflicts of interest.

\section{References}

[1] I. Harris, Y. Wang, and H. Wang, "ICT in multimodal transport and technological trends: unleashing potential for the future," International Journal of Production Economics, vol. 159, no. 1, pp. 88-103, 2015.

[2] S. Mathaba, M. Adigun, J. Oladosu, and O. Oki, "On the use of the Internet of Things and Web 2.0 in inventory management," Journal of Intelligent \& Fuzzy Systems, vol. 32, no. 4, pp. 3091-3101, 2017.

[3] C. Lloyd, T. Pötsch, S. Yi et al., "Resources in logistics-a multidisciplinary challenge," IFAC Proceedings Volumes, vol. 46, no. 24, pp. 449-455, 2013.

[4] G. Cabrera, P. A. Miranda, E. Cabrera et al., "Solving a novel inventory location model with stochastic constraints and (R, s, S) inventory control policy," Mathematical Problems in Engineering, vol. 2013, Article ID 670528, 12 pages, 2013.

[5] Y. Ma, X. Song, J. Wang, and Z. Xiao, "A practical infrastructure for real-time simulation across timing domains," Mathematical Problems in Engineering, vol. 2015, Article ID 163845, 12 pages, 2015.

[6] M. G. Speranza, "Trends in transportation and logistics," European Journal of Operational Research, vol. 264, no. 3, pp. 830-836, 2016.

[7] S. M. Disney, I. Farasyn, M. Lambrecht, D. R. Towill, and W. V. de Velde, "Taming the bullwhip effect whilst watching customer service in a single supply chain echelon," European Journal of Operational Research, vol. 173, no. 1, pp. 151-172, 2006.

[8] P. Ignaciuk and A. Bartoszewicz, "LQ optimal sliding-mode supply policy for periodic-review perishable inventory systems," Journal of The Franklin Institute, vol. 349, no. 4, pp. 1561-1582, 2012.

[9] P. Ignaciuk and A. Bartoszewicz, "Discrete sliding-mode control of inventory systems with deteriorating stock and remote supply source," Control Engineering and Applied Informatics, vol. 14, no. 1, pp. 14-21, 2012.

[10] K. Xu and P. T. Evers, "Managing single echelon inventories through demand aggregation and the feasibility of a correlation matrix," Computers \& Operations Research, vol. 30, no. 2, pp. 297-308, 2003.

[11] M. E. Seliaman, "Optimizing the two-stage supply chain inventory model with full information sharing and two backorders costs using hybrid geometric-algebraic method," Journal of Optimization, vol. 2013, Article ID 519180, 5 pages, 2013.

[12] L. Sun and Y. Zhou, "A knowledge-based tree-like representation for inventory routing problem in the distribution system of oil products," Procedia Computer Science, vol. 112, pp. 1683-1691, 2017.

[13] X. Shi, "Efficiency bounds for two-stage production systems," Mathematical Problems in Engineering, vol. 2018, Article ID 2917537, 9 pages, 2018.

[14] P. Ignaciuk, "Dead-time compensation in continuous-review perishable inventory systems with multiple supply alternatives," Journal of Process Control, vol. 22, no. 5, pp. 915-924, 2012.

[15] D. Simchi-Levi and Y. Zhao, "Performance evaluation of stochastic multi-echelon inventory systems: a survey," Advances in Operations Research, vol. 2012, Article ID 126254, 34 pages, 2012.

[16] P. Ignaciuk, "LQ optimal and robust control of perishable inventory systems with multiple supply options," IEEE Transactions on Automatic Control, vol. 58, no. 8, pp. 21082113, 2013.

[17] A. Gunasekaran, N. Subramanian, and S. Rahman, "Supply chain resilience: role of complexities and strategies," International Journal of Production Research, vol. 53, no. 23, pp. 6809-6819, 2015.

[18] Z. Liu, W. Deng, and G. Chen, "Analysis of the optimal resource allocation for a tandem queueing system," Mathematical Problems in Engineering, vol. 2017, Article ID 5964272, 10 pages, 2017.

[19] J. D. Sterman, Business Dynamics: Systems Thinking and Modeling for a Complex World, McGraw-Hill, New York, NY, USA, 2000.

[20] J. W. Forrester, Industrial Dynamics, Cambrige MIT Press, Cambridge, MA, USA, 1963.

[21] D. R. Towill, "Supply chain dynamics," International Journal of Computer Integrated Manufacturing, vol. 4, no. 4, pp. 197-208, 1992.

[22] D. R. Towill and A. Del Vecchio, "The application of filter theory to the study of supply chain dynamics," Production Planning \& Control, vol. 5, no. 1, pp. 82-96, 1994.

[23] C. E. Riddalls, S. Bennett, and N. S. Tipi, "Modelling the dynamics of supply chains," International Journal of Systems Science, vol. 31, no. 8, pp. 969-976, 2000.

[24] C. E. Riddalls and S. Bennett, "The stability of supply chains," International Journal of Production Research, vol. 40, no. 2, pp. 459-475, 2002.

[25] K. Singha, J. Buddhakulsomsiri, and P. Parthanadee, "Mathematical model of $(\mathrm{R}, \mathrm{Q})$ inventory policy under limited storage space for continuous and periodic review policies with backlog and lost sales," Mathematical Problems in Engineering, vol. 2017, Article ID 4391970, 9 pages, 2017.

[26] K. Hoberg, J. R. Bradley, and U. W. Thonemann, "Analyzing the effect of the inventory policy on order and inventory variability with linear control theory," European Journal of Operational Research, vol. 176, no. 3, pp. 1620-1642, 2007. 
[27] P. Ignaciuk, "Discrete inventory control in systems with perishable goods-a time-delay system perspective," IET Control Theory \& Applications, vol. 8, no. 1, pp. 11-21, 2014.

[28] P. Ignaciuk, "Discrete-time control of production-inventory systems with deteriorating stock and unreliable supplies," IEEE Transactions on Systems, Man, and Cybernetics: Systems, vol. 45, no. 2, pp. 338-348, 2015.

[29] Y. Wei, H. Wang, and F. Chen, "Exploring the impact of network structure and demand collaboration on the dynamics of a supply chain network using a robust control approach,” Mathematical Problems in Engineering, vol. 2015, Article ID 102727, 13 pages, 2015.

[30] F. Blanchini, F. Rinaldi, and W. Ukovich, "A network design problem for a distribution system with uncertain demands," SIAM Journal on Optimization, vol. 7, no. 2, pp. 560-578, 1997.

[31] F. Blanchini, S. Miani, and W. Ukovich, "Control of production-distribution systems with unknown inputs and system failures," IEEE Transactions on Automatic Control, vol. 45, no. 6, pp. 1072-1081, 2000.

[32] M. W. Braun, D. E. Rivera, M. E. Flores, W. M. Carlyle, and K. G. Kempf, "A model predictive control framework for robust management of multi-product, multi-echelon demand networks," Annual Reviews in Control, vol. 27, no. 2, pp. 229-245, 2003.

[33] C. I. Papanagnou and G. D. Halikias, "Supply-chain modelling and control under proportional inventory-replenishment policies," International Journal of Systems Science, vol. 39, no. 7, pp. 699-711, 2008.

[34] P. Ignaciuk and A. Bartoszewicz, "Linear-quadratic optimal control strategy for periodic-review inventory systems," Automatica, vol. 46, no. 12, pp. 1982-1993, 2010.

[35] M. Ortega and L. Lin, "Control theory applications to the production-inventory problem: a review," International Journal of Production Research, vol. 42, no. 11, pp. 2303-2322, 2004.

[36] H. Sarimveis, P. Patrinos, C. D. Tarantilis, and C. T. Kiranoudis, "Dynamic modeling and control of supply chain systems: a review," Computers \& Operations Research, vol. 35, no. 11, pp. 3530-3561, 2008.

[37] S. Axsäter, Inventory Control, Springer International Publishing, New York, NY, USA, 2015.

[38] A. Zemzam, M. E. Maataoui, M. Hlyal, J. E. Alami, and N. E. Alami, "Inventory management of supply chain with robust control theory: literature review," International Journal of Logistics Systems and Management, vol. 27, no. 4, pp. 438-465, 2017.

[39] D. Ivanov, S. Sethi, A. Dolgui, and B. Sokolov, "A survey on control theory applications to operational systems, supply chain management, and industry 4.0," Annual Reviews in Control, vol. 46, pp. 134-147, 2018.

[40] D. Bertsimas and A. Thiele, "A robust optimization approach to inventory theory," Operations Research, vol. 54, no. 1, pp. 150-168, 2006.

[41] D. C. Tarraf and D. Bauso, "Finite alphabet control of logistic networks with discrete uncertainty," Systems \& Control Letters, vol. 64, pp. 20-26, 2014.

[42] P. Ignaciuk, "Nonlinear inventory control with discrete sliding modes in systems with uncertain delay," IEEE Transactions on Industrial Informatics, vol. 10, no. 1, pp. 559-568, 2014.

[43] K. K. Movahed and Z.-H. Zhang, "Robust design of (s, S) inventory policy parameters in supply chains with demand and lead time uncertainties," International Journal of Systems Science, vol. 46, no. 12, pp. 258-268, 2015.

[44] S. Cannella, R. Dominguez, B. Ponte, and J. M. Framinan, "Capacity restrictions and supply chain performance: modelling and analysing load-dependent lead times," International Journal of Production Economics, vol. 204, pp. 264-277, 2018.

[45] C. Danielson, F. Borrelli, D. Oliver, D. Anderson, and T. Phillips, "Constrained flow control in storage networks: capacity maximization and balancing," Automatica, vol. 49, no. 9, pp. 2612-2621, 2013.

[46] M. Burger, C. De Persis, and F. Allgower, "Dynamic pricing control for constrained distribution networks with storage," IEEE Transactions on Control of Network Systems, vol. 2, no. 1, pp. 88-97, 2015.

[47] N. Zhao, M. Xia, C. Mi, Z. Bian, and J. Jin, "Simulation-based optimization for storage allocation problem of outbound containers in automated container terminals," Mathematical Problems in Engineering, vol. 2015, Article ID 548762, 14 pages, 2015.

[48] A. D. P. Lima, F. W. de Mascarenhas, and E. M. Frazzon, "Simulation-based planning and control of transport flows in port logistic systems," Mathematical Problems in Engineering, vol. 2015, Article ID 862635, 12 pages, 2015.

[49] T. de Kok, C. Grob, M. Laumanns, S. Minner, J. Rambau, and K. Schade, "A typology and literature review on stochastic multi-echelon inventory models," European Journal of Operational Research, vol. 269, no. 3, pp. 955-983, 2018.

[50] N. Shi, H. Song, and W. B. Powell, "The dynamic fleet management problem with uncertain demand and customer chosen service level," International Journal of Production Economics, vol. 148, pp. 110-121, 2014.

[51] C.-M. Chen, H.-H. Chiu, Y.-P. Chi, and S.-C. Wu, "Does uncertain demand affect service quality?" International Journal of Hospitality Management, vol. 46, pp. 76-78, 2015.

[52] M. Bucciarelli, S. Paoletti, and A. Vicino, "Optimal sizing of energy storage systems under uncertain demand and generation," Applied Energy, vol. 225, pp. 611-621, 2018.

[53] K. D. Cattani, F. R. Jacobs, and J. Schoenfelder, "Common inventory modeling assumptions that fall short: arborescent networks, Poisson demand, and single-echelon approximations," Journal of Operations Management, vol. 29, no. 5, pp. 488-499, 2011.

[54] J. Dejonckheere, S. M. Disney, M. R. Lambrecht, and D. R. Towill, "Measuring and avoiding the bullwhip effect: a control theoretic approach," European Journal of Operational Research, vol. 147, no. 3, pp. 567-590, 2003.

[55] P. Ignaciuk and L. Wieczorek, "Application of continuous genetic algorithms for optimization of logistic networks governed by order-up-to inventory policy," International Journal of New Computer Architectures and Their Applications, vol. 7, no. 1, pp. 29-36, 2017. 


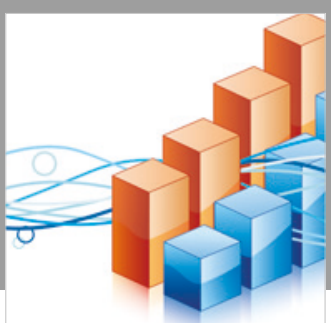

Advances in

Operations Research

\section{-n-m}
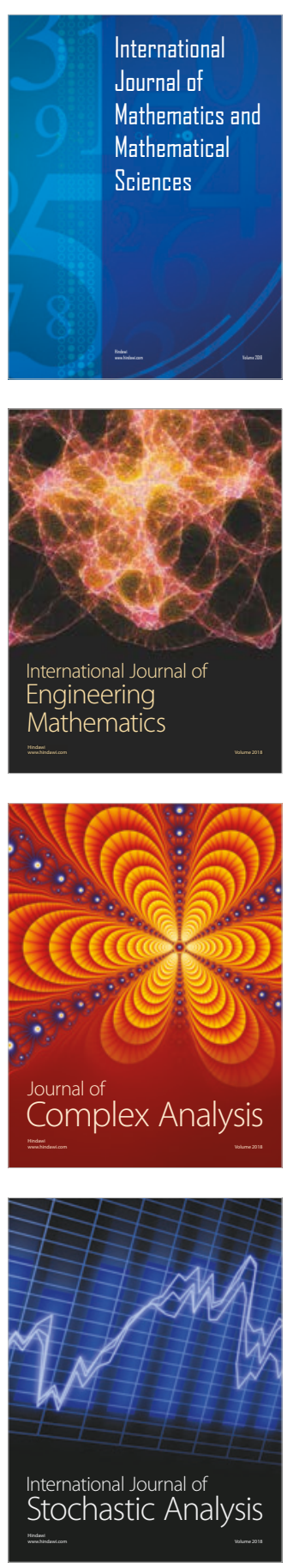
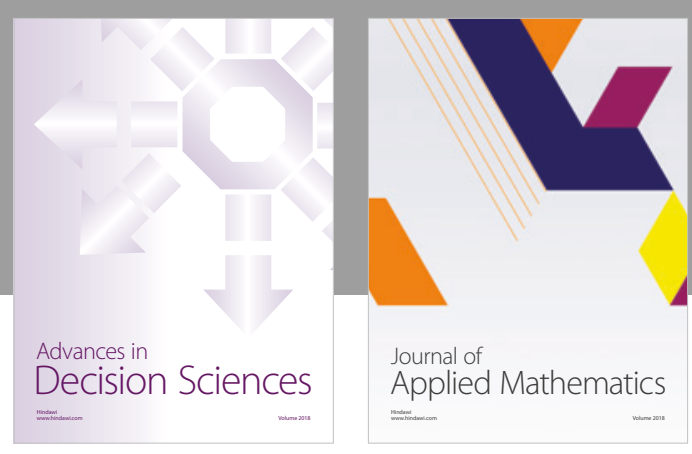

Journal of

Applied Mathematics
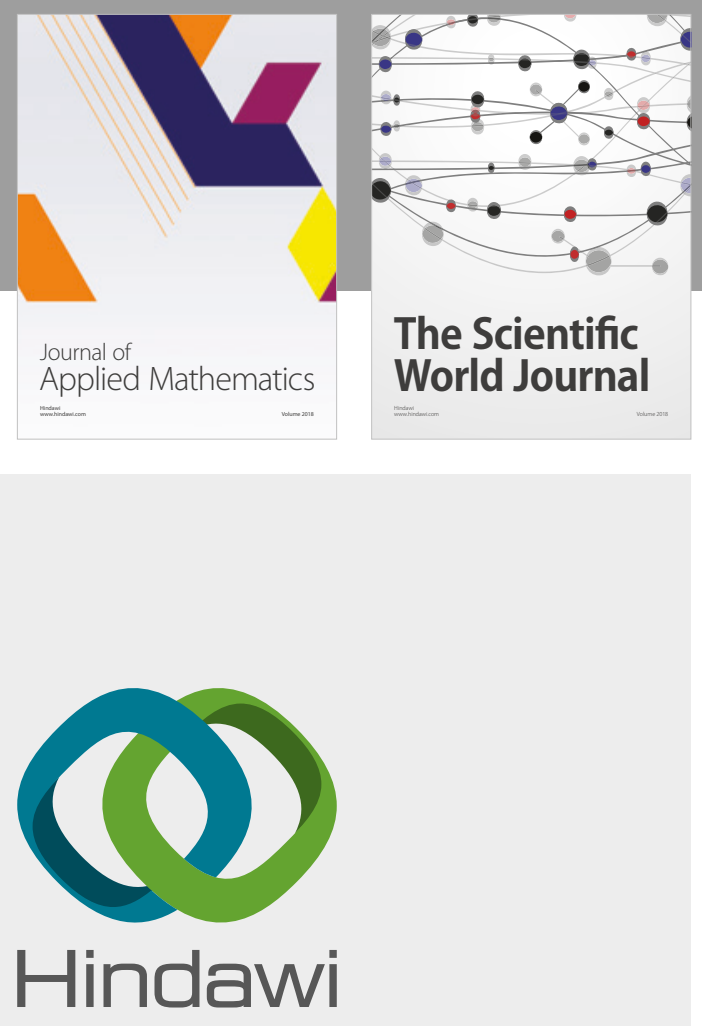

Submit your manuscripts at

www.hindawi.com

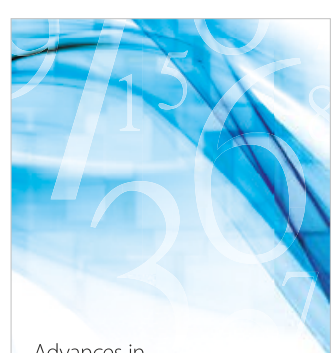

Advances in
Numerical Analysis
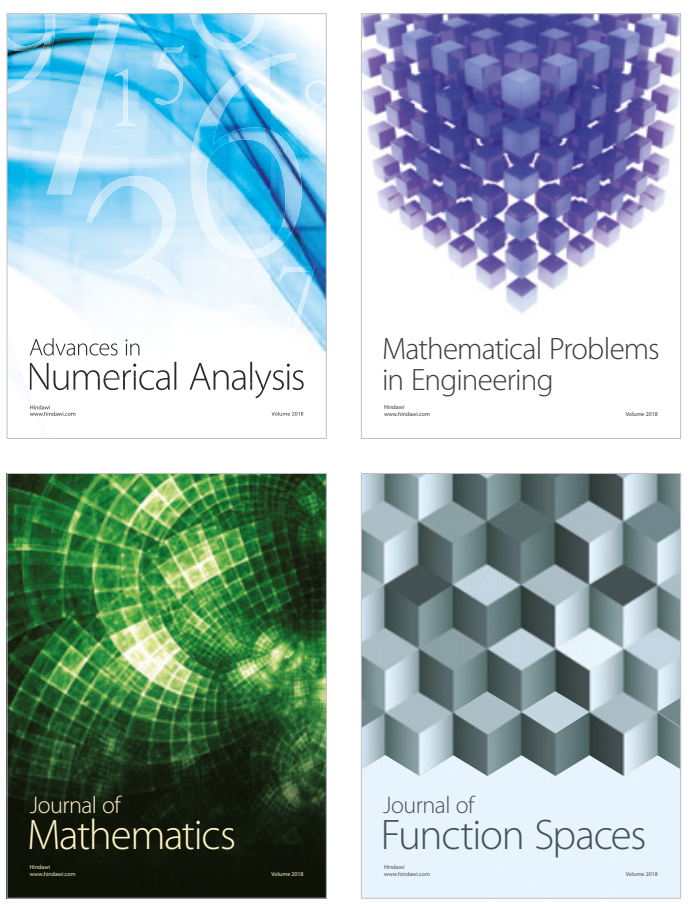

Mathematical Problems in Engineering

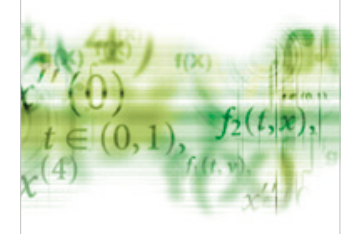

International Journal of

Differential Equations

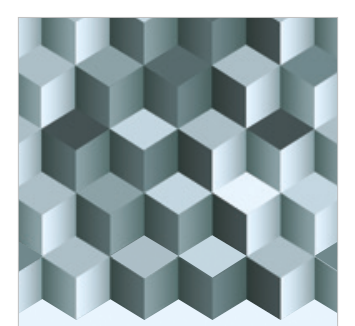

Journal of

Function Spaces

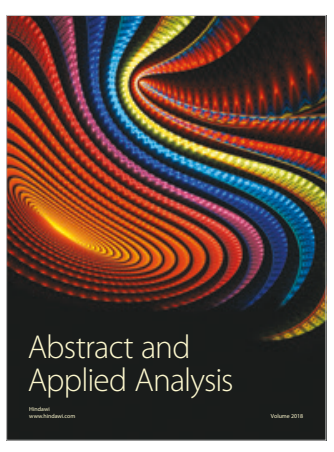

The Scientific

World Journal

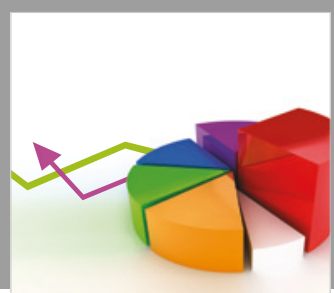

Journal of

Probability and Statistics
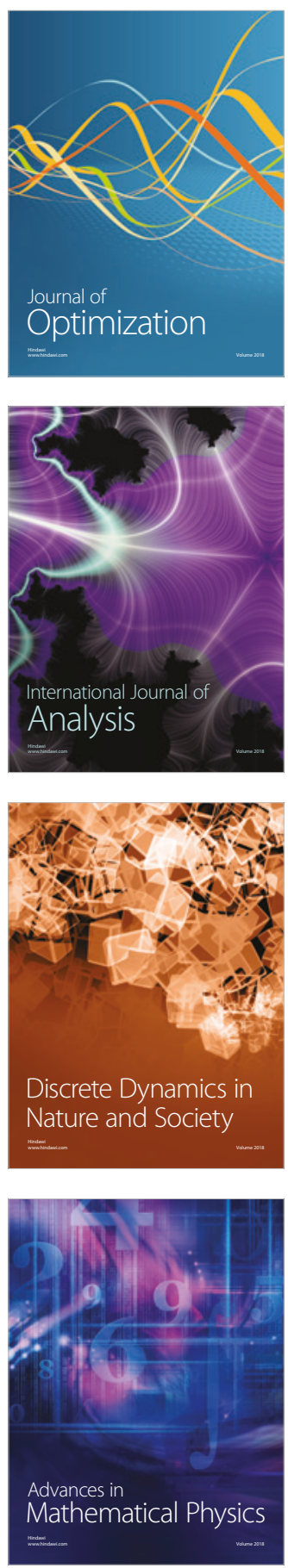